\title{
Landslide Warning System Using ZigbeeAnd GPS
}

\author{
Y.Srinivas, \\ M.Tech Project Scholar, \\ K. Raghava Rao \\ Professor in CSE \\ Department of Electronics and Computer Engineering, Vaddeswaram KL University, Guntur
}

\begin{abstract}
In this project we are going to study about Landslide warning system, as the occurrence of landslides is a big loss for human life and property. We can't stop the natural causes but we can be alert before they occur. So for alerting people from landslides we use this technique.

In this design we have used three sensors of Angle sensor which gives the readings of slope angle if there is any movement in landslide and we have Liquid level sensor it collects the depth of water at the mountains. Temperature sensor gives the changes in the temperature. These all nodes of sensors are connected to the LPC 2148 ARM processor for collection of data. As the data is collected then GPS gives latitude and longitude and all the readings are given to zigbee for transmission.

As we obtain the information at the receiver side by LCD display at receiver station or by SMS we can alert the people and save lives and property. Thus this project is very important as we use it in our real time purpose for saving lives and property. This design combines of GSM wireless communication technology and Wireless Sensor Network.
\end{abstract}

Key words: Zigbee, GPS, GSM

\section{INTRODUCTION}

In order to reduce and prevent the damage of landslides, landslide monitoring is very important to the prediction and estimation of the landslide hazard and prevention. Landslide monitoring is an important topic related at the hill slides. Landslides are geological phenomena causing significant loss of life and loss of properties in damages each year in many countries. Therefore technology has to be developed to capture relevant signals with minimum monitoring delay. Wireless sensors are one of the technologies that can quickly respond to rapid changes of data and send the sensed data to the receiver section in areas where cabling is not available.

Wireless sensor network (WSN) technology has the capability of quick capturing, processing, and transmission of required data in real-time with high resolution. However, it has its own limitations such as relatively low amounts of battery power and low memory availability compared to many existing technologies. It does, though, have the advantage of deploying sensors in hostile environments with a minimum of maintenance. This fulfills a very important need for any real time monitoring, especially in hazardous or remote scenarios. GPS is being increasingly used also for automated continuous monitoring of landslides and avalanches. For such GPS-based deformation monitoring systems, the accuracy, availability, reliability and integrity of the positioning solutions heavily depend on the number and geometric distribution of satellites being tracked. It is composed of a series of sensors, of easy and rapid installation, which can be positioned directly upon the landslide body, or in the immediate surroundings, to provide continuous data on the landslide activity. The SMS technology also enables the user to dialogue with the system, to obtain information on the monitoring operations, either by means of SMS messages. They cause considerable damage to highways, railways, waterways and pipelines. They commonly occur with other major natural disasters such as earthquakes), volcanic activity, and floods caused by heavy rainfall. In many cases, expanded development and human activities, such as modified slopes and deforestation, can increase the incidence of landslide disasters.

Problem Definition: Here we have used the Zigbee, GSM and GPS modules as earlier we have used GSM and Zigbee. As by using GPS we can have the accurate location of the place.

\section{DESIGN OF LANDSLIDE WARNING SYSTEM}

In this we have the modules of GPS, Zigbee, GSM and three sensors. The sensors used are Angle sensor, Water level sensor and Temperature sensor. Water level sensors and angle sensors will convert water depth, hillsides displacement and temperature level to electrical signals, then electrical signals from the sensors will be voltage converted, and amplified in signal disposal circuit at last signals are sent to processor. This design adopts high precision angle sensor SCA60C produced by VTI to detect the mountain angle. SCA60C is single axis angle sensor, its measuring range is +90 degrees, $+5 \mathrm{v}$ power supply, the output voltage is proportional to the hill slide displacement, its output range is $0.5-4.5 \mathrm{v}$, the capability of resistant impact is strong and have low power consumption. SCA60C is actually a accelerometer, it consists of a silicon micro sensor and 
signal processing chip, through measuring the earth's gravity in the direction of measurement, then convert to angle. If the landslides along the direction of arrow on the chips have acceleration, the output value increases.

Hear zigbee and GPS are connected to processor using serial communication. The message from transmitter is send through transmitter zigbee device and received by receiver side zigbee and the information is given through sms by GSM connected at receiver. We have LCD at both transmitter and receiver to observe the readings directly at receiver station without sms. This is the overall function of a components in block diagram.

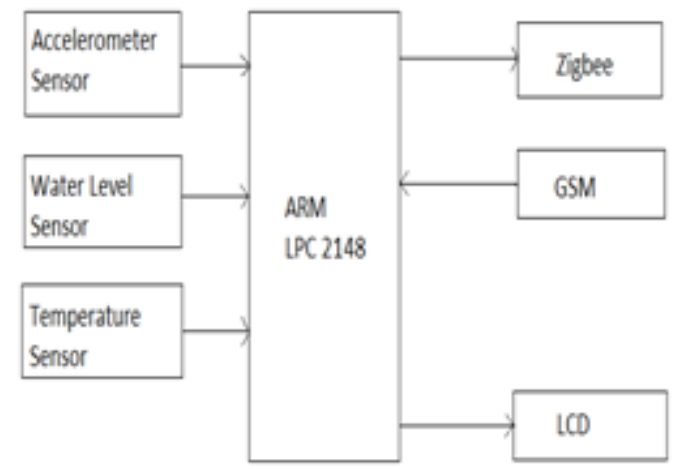

Fig1.Block Diagram of Transmitter Section

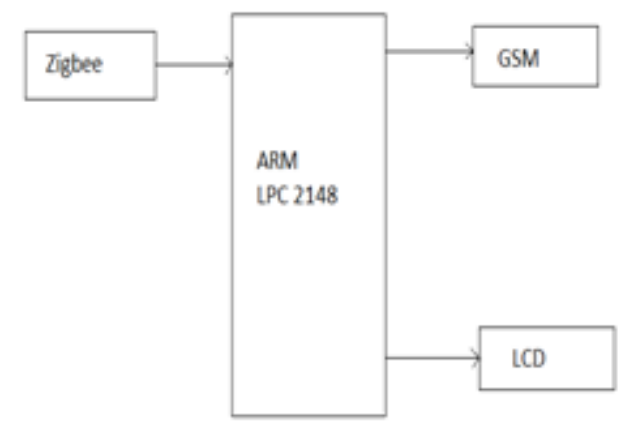

Fig2: Block Diagram of Receiver Section

III. SENSORS USED

Hear we have used three sensors for efficient and effective working of our landslide warning system. They are 1) MEMS(Accelerometer), 2) Water level sensor, 3) Temperature sensor.

MEMS is Micro Electro Mechanical Systems, this sensor senses if there is any movement in the angle of sensor it sends the data of movement of angle to receiver side. MEMS is the integration of mechanical elements, sensors, actuators, and electronics on a common silicon substrate through micro fabrication technology. It is placed on the land if any movement occurs then sensor senses it and sends information so that we can be alert at that area.

Water level sensor is used to measure the water level in hill stations under water level increases if any disturbance in hills. So we use water level sensor to measure water level it is connected using transistor, a wire of $5 \mathrm{v}$ power supply is immersed in water then a scale with levels $1,2,3$ are immersed in water as water level increases it shows at which level water is, so that we can be alert.

Temperature sensor is used to measure temperature level at the hill stations as hill slides may occur due to volcanic eruptions alsoso as temperature increases the sensor senses and gives information to processor. We used IC called LM35 as a temperature sensor. LM35 senses temperature and converted to digital form using $\mathrm{ADC}$ and given to processor.

\section{ZIGBEE}

Zigbee is the main hardware module used in this paper for communication purpose. We have two zigbee modules one at transmitter side, another at receiver side. By using this zigbee we can communicate data of high speed and more amounts of data can be sent through zigbee. We don't need any external signal for this communication, so we can use this zigbee modules anywhere even in forests, hill stations etc. in this we have peer to peer communication. One zigbee can communicate with any other zigbee present in the surrounding area. The transmitter zigbee transmits the data obtained from transmitter side processor it transmits without having delay. Then receiver side zigbee receives the signal and automatically receives data transmitted from 
transmitter. In this we don't have data loss so it is safe and useful by using zigbee modules. In this zigbee there is no need of human intervention to send or receive data it automatically sends and receives data. In this we use IEEE 802.15.4 protocol stack.

\section{GPS}

The Global Positioning System (GPS) is a worldwide radio-navigation system. This module is used to show the accurate location of the landslide detection device placed, so as to know at which place the landslide occurs. This GPS module is installed in transmitter section and if any disturbance occurs in sensors attached then processor automatically send the information of location of module to receiver side this helps us to locate the hazardous areas. This GPS module calculates its position by measuring its distance from itself and other satellites and obtains the location in latitude and longitude. As in hill stations we don't know where the landslide occurs accurately we use this GPS to know location accurately so that we can alert people at that area and save them.

\section{MICROCONTROLLER}

Hear we used LPC2148 microcontroller.The LPC2148 microcontroller is based on a 32 bit ARM7TDMI-S CPU with real-time emulation and embedded trace support, that combines the microcontroller with embedded high speed flash memory up to $512 \mathrm{~kb}$. A 128-bit wide memory interface and a unique accelerator.

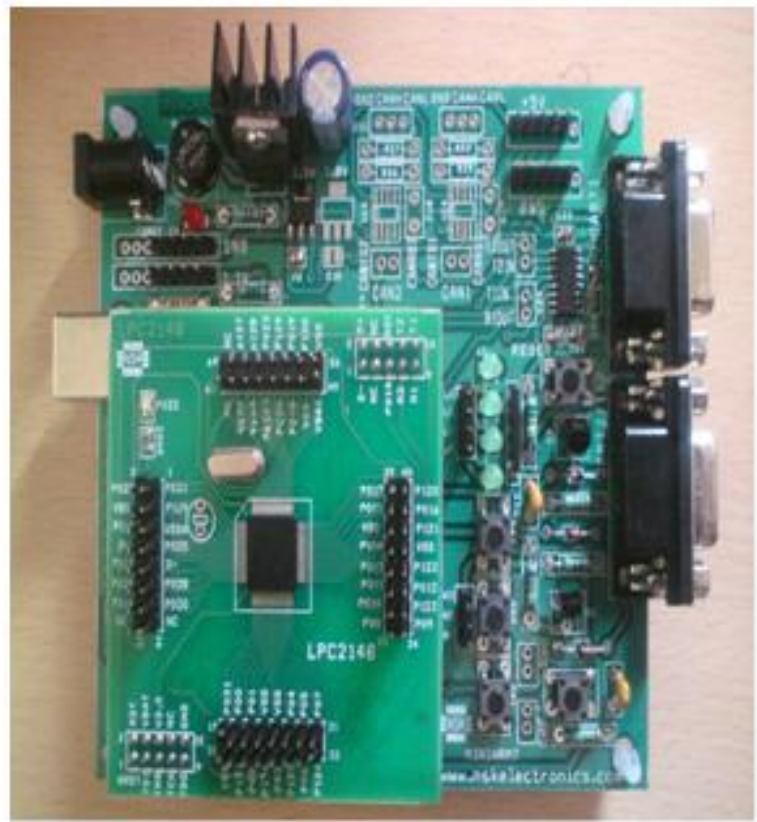

Fig 3.ARM (Advanced RISC Machine)

To this microcontroller Zigbee and GPS modules are attached. Microcontroller performs the operations of collecting data and transferring data from sensors and GPS to Zigbee, and display the outputs on LCD screen.

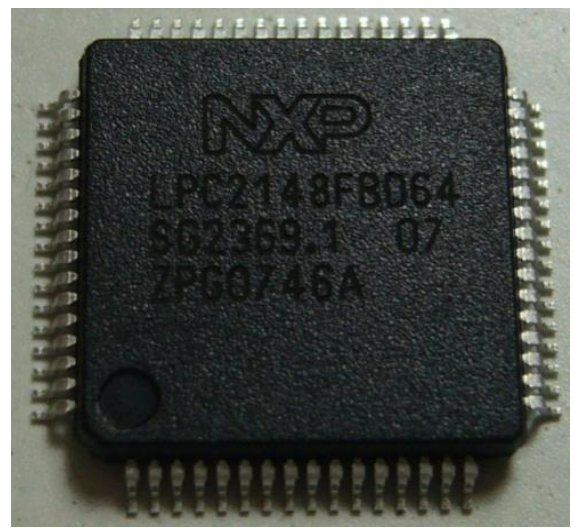

Fig4. LPC2148 microcontroller 


\section{CONCLUSION \& FUTURE WORK}

We have designed the real time system for landslide detection. This system is developed using LPC 2148 ARM processor. The main components used in this system are Zigbee, GPS, GSM. We have attached three sensors like water level sensor, mems, temperature sensor. In the transmitter section we have sensors connected to ARM processor if any sensor detected any disturbance zigbee transmits signal to receiver zigbee and it dispalys in LCD at receiver and by GSM we got the message.

We have done some testing on working of the system in we have observed landslide detection in fig 3 and we obtain message to phone and displayed at LCD in receiver section. In fig 4, 5, 6 we have observed water level change and message received at receiver section. In fig 7, 8, 9 we have observed change in temperature if it reaches $45^{*}$ degrees we will receive message and displays over temperature at receiver section.

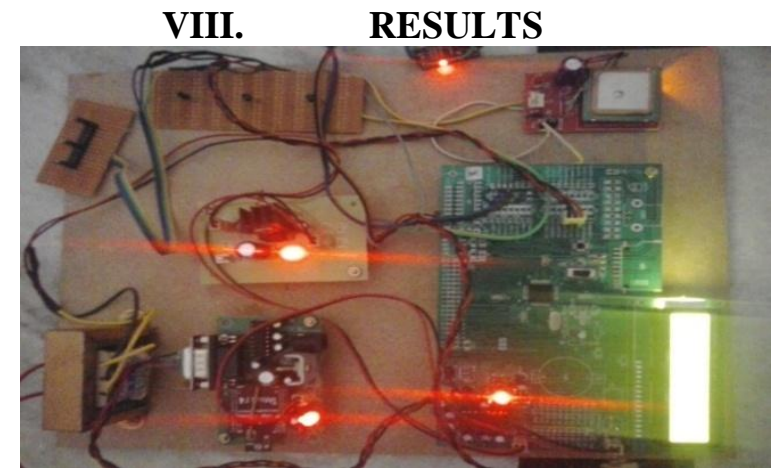

Fig1: Transmitter Section

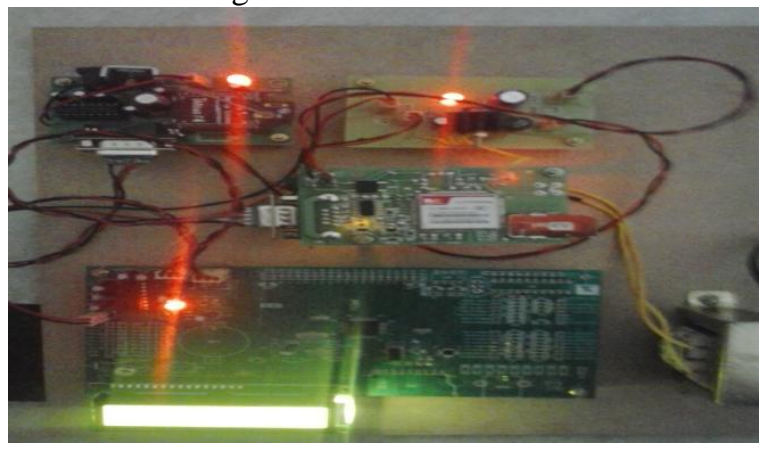

Fig2: Receiver Section

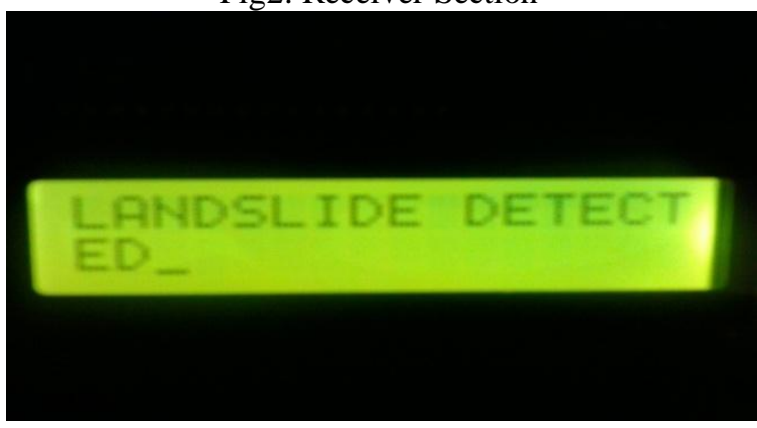

Fig3: MEMS Output

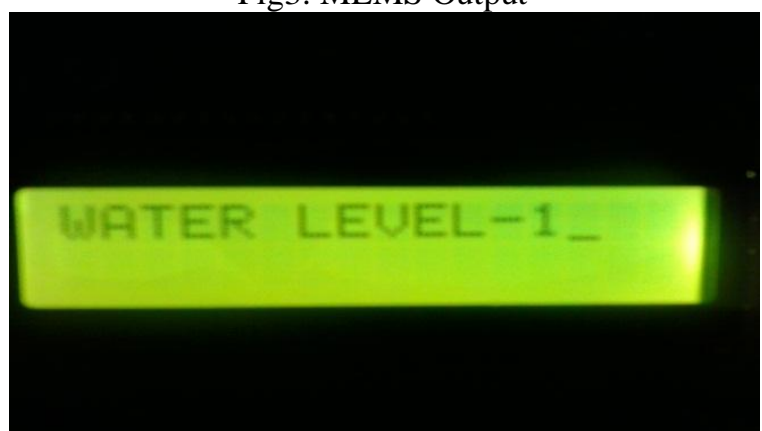

Fig4: Water Level Sensor at level 1 


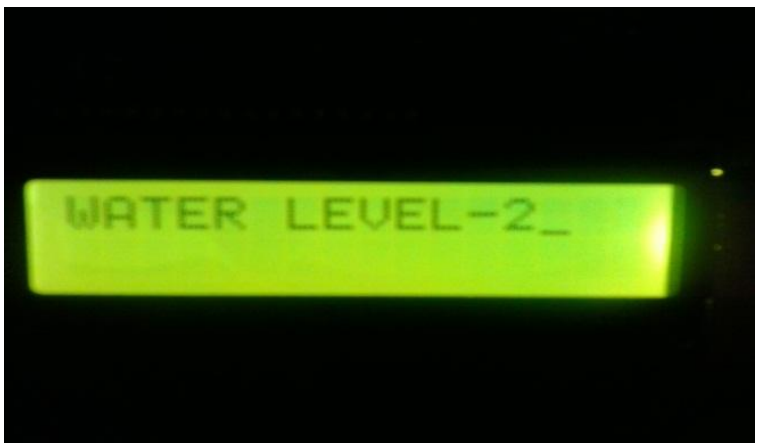

Fig5: Water Level Sensor at level 2

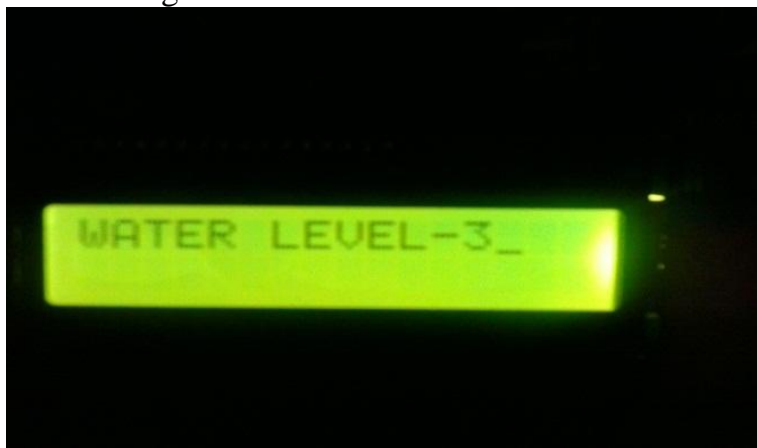

Fig6: Water Level Sensor at level 3

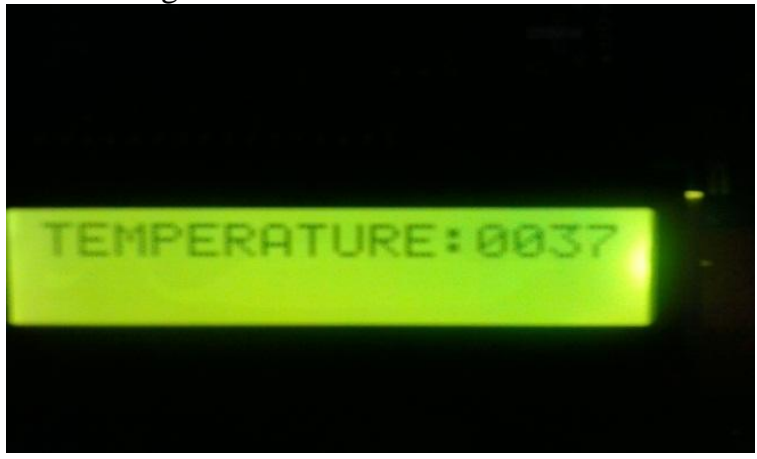

Fig7: Temperature Sensor at 37*

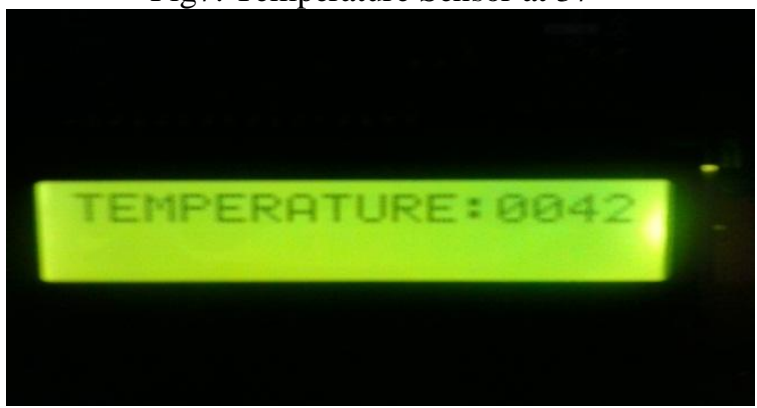

Fig8: Temperature Sensor at 42*

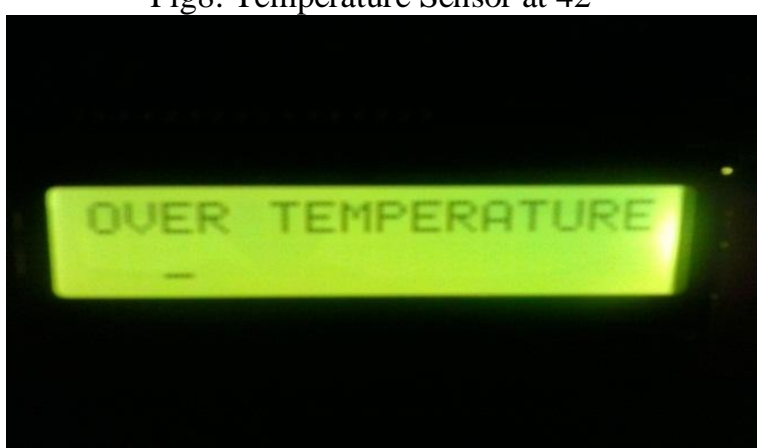

Fig9: Temperature Sensor above 45* 


\section{REFERENCES}

[1] Charles M. Hammond, P.G., C.E.G, L.H.G, "Geology in Landslide Engineering", North American Landslides Conference presentations held at Vail, Colorado, June 3-8, 2007.

[2] Su Baishun, Pang Zhengduo, MengGuoying "Design of the mine gas sensor based on Zigbee", Proceedings of the Third International Symposium on Computer Science and Computational Technology(ISCSCT '10) Jiaozuo, P. R. China, 14-15,August 2010.

[3] Vern Singhroy, Hiroshi Ohkura, Nancy Glenn, "Earth Observation for Landslide Assessment", 2002, IEEE.

[4] AbishekThekkeyilKunnath, Maneesha V. Ramesh, "Integrating Geophone Network to Real-Time Wireless Sensor Network System for Landslide Detection", 2010 First International Conference on Sensor Device Technologies and Applications

[5] C. Tarantino, P. Blonda, G. Pasquariello, "Application of change detection techniques formonitoring man-induced landslide causal factors", 2004, IEEE.

[6] SitiKhairunniza-Bejol, Maria Petrou2t and Athanassios Ganas3, "Landslide Detection Using a Local Similarity Measure", 2006, IEEE.

[7] Peng Liu, Wangdong Qi, En Yuan, Yasong Zhu, Han Wang, "Ground Displacement Measurement by Radio Interferometry Ranging for Landslide Early Warning", 2011, IEEE.

[8] Andreas Terzis, AnnalingamAnandarajah, Kevin Moore, "Slip Surface Localization in Wireless Sensor Networks for Landslide Prediction”, IPSN'06, April 19-21, 2006, Nashville, Tennessee, USA.

[9] Leonardo Zan, Gilberto Latini, EvasioPiscina, Giovanni Polloni, PieramelioBaldelli, "Landslides Early Warning Monitoring System", 2002, IEEE.

[10] Mingsheng Liao, Lu Zhang, TimoBalz, "Post-earthquake landslide detection and early detection of landslide prone areas using SAR", 2009, IEEE.

[11] Maneesha V. Ramesh, "Real-time Wireless Sensor Network for Landslide Detection", 2009 Third International Conference on Sensor Technologies and Applications.

[12] Andreas Terzis, AnnalingamAnandarajah, Kevin Moore, I-Jeng Wang, "Slip Surface Localization in Wireless Sensor Networks for Landslide Prediction”, IPSN'06, April 19-21, 2006, Nashville, Tennessee, USA.

[13] Zhuan Chen, Liping Deng, "The Design of Landslide Monitoring and Early-warning System in Opencast Based on Non-metric Digital Camera”, 2012, Science of Surveying and Mapping.

\section{ABOUT AUTHORS}

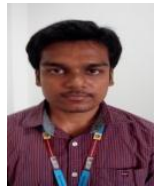

Y.Srinivas, of M Tech ECM, He is doing M Tech from 2012-2014 at K L University. He has done B Tech on Electronics and Communication Engineering. In B Tech he has done a project on "Fall Detection of patients using 3-Axis Accelerometer. He is continuing his researchon sensors and embedded system.

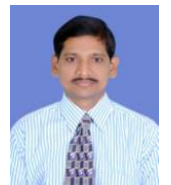

K.Raghava Rao, Professor in CSE, working in the dept. of ECM, KL University. He is having 15 years of experience in teaching for UG and PG engineering students and 3 years of Software Development experience in Singapore. He received B.E(CSE) from MG state University, M.Tech(CSE) from RVP University, Udaipur and Ph.D(CSE) from Mahatma Gandhi University (KasiVidyapeeth), Varanasi, in the years 1995, 2005 and 2009 respectively. He published several papers in national \& international conferences and journals. He published 3 text books. Currently he is carrying a DST funded project in the area of Wireless sensor networks-sensor web enablement. His research interests are Wireless sensor networks, Embedded Sensor networks and Sensor Web Services. He received paper award from DST Secretary and Best Teacher award from KL University He is also member of several technical organizations. 